\title{
Nonlinear vibrational analogue of coherent output coupler for atoms in Bose-Einstein condensate
}

\author{
Yu.A.Kosevich ${ }^{\dagger}$ \\ †yukosevich@gmail.com
}

\author{
Semenov Institute of Chemical Physics RAS, Kosygin str. 4, 119991 Moscow, Russia
}

\begin{abstract}
The analogies between coherent matter waves in Bose-Einstein condensates of atomic gases and coherent photons have been discussed in connection with possible realization of an "atom laser". Theoretical discussions of the atom laser have considered the case in which atoms are fed into and coupled out of the condensate continuously. Here we discuss the analogy between the output coupler for the trapped coherent matter waves and collective nonlinear energy transfer in a system of three coupled pendulums with variable parameters. One pendulum in the system is weakly coupled through a spring with the pair of pendulums, which are tightly bound through a common string. We show that self-sustained oscillations of the energy distribution between the weakly coupled and tightly bound subsystems can occur. In our simulations, the coherent fraction of the total mechanical energy of the system, which is periodically transferred to the weakly linked subsystem, can be of the order of 1/4000 and even smaller. These periodic oscillations in the energy distribution are similar, and their time evolution is described by similar functions of time, to the quantum Rabi oscillations in the populations of the trapped and untrapped (out-coupled) states in the coherent output coupler for atoms in Bose-Einstein condensate. The described effects can be used for the coherent control of the transfer of mechanical energy on the micro- and nano-scales.
\end{abstract}

Keywords: vibration energy transfer, mechanical energy, coupled pendulums.

\section{Introduction}

Realization of Bose-Einstein condensation in atomic gases provides samples of atoms with a macroscopic population in the ground state of the system. This population forms a coherent matter wave and is described by a macroscopic wave function, which is the solution of the nonlinear Gross-Pitaevskii equation. The analogies between coherent matter waves and coherent photons have been discussed in connection with possible realization of an "atom laser" [1].

Theoretical discussions of the atom laser have considered the case in which atoms are fed into and coupled out of the "lasing mode" continuously. The output coupler for Bose condensed atoms in a magnetic trap has been demonstrated, in which Bose condensate in a superposition of trapped and untrapped hyperfine states was created with short pulses of radio-frequency radiation, and the fraction of out-coupled atoms was adjusted between $0 \%$ and $100 \%$ by varying the amplitude of radiation [2].

Here we discuss the analogy between the output coupler for the trapped coherent matter waves and the nonlinear coherent energy transfer in a system of three coupled mechanical oscillators (pendulums) with variable parameters. One pendulum in the considered system is weakly linked with a pair of pendulums that is tightly bound through a common string. We show that self-sustained oscillations of the energy distribution between the weakly linked and the tightly bound subsystems can occur. In our simulations, the coherent fraction of the total vibration energy of the system, which is periodically given to the weakly linked subsystem, can be less than $1 / 4000$. These oscillations of the energy distribution and coherent energy transfer are similar, and their time evolution is described by similar functions of time, to the quantum Rabi oscillations in the populations of the trapped and untrapped (out-coupled) states, which were observed in the coherent output coupler for sodium atoms in Bose-Einstein condensate [2].

\section{Model}

We start from the following Lagrangian:

$$
\begin{gathered}
L=\frac{1}{2}\left[m_{1} l_{1}^{2}\left(\dot{\varphi}_{1}\right)^{2}+m_{2} l_{2}^{2}\left(\dot{\varphi}_{2}\right)^{2}+\left(m_{2}+m_{3}\right)\left(\dot{l}_{2}\right)^{2}\right]- \\
-g\left[m_{1} l_{1}\left(1-\cos \varphi_{1}\right)+m_{2}\left(l_{20}-l_{2} \cos \varphi_{1}\right)+m_{3}\left(l_{2}-l_{20}\right)\right]- \\
-k_{12}\left(l_{1} \sin \varphi_{1}-l_{2} \sin \varphi_{2}\right)^{2}
\end{gathered}
$$

where $\varphi_{1,2}$ and $l_{1,2}$ are the deflection angles and string lengths of pendulums 1 and $2, l_{20}$ is the initial string length of pendulum 2 in the pendulum pair of particles 2 and $3, k_{12}$ is the coupling constant between particles 1 and $2, g$ is gravity acceleration. The corresponding equations of motion are:

$$
\begin{aligned}
& \ddot{\varphi}_{1}+g \sin \varphi_{1} / l_{1}+k_{12} \cos \varphi_{1}\left(\sin \varphi_{1}-l_{2} \sin \varphi_{2} / l_{1}\right) \frac{1}{m_{1}}=0, \\
& \ddot{\varphi_{2}}+g \sin \varphi_{2} / l_{2}+ \\
& +2 \dot{l}_{2} \dot{\varphi}_{2} / l_{2}+k_{12} \cos \varphi_{2}\left(\sin \varphi_{2}-l_{1} \sin \varphi_{1} / l_{2}\right) \frac{1}{m_{2}}=0,
\end{aligned}
$$




$$
\begin{aligned}
\ddot{l}_{2}\left(m_{2}\right. & \left.+m_{3}\right)+g\left(m_{3}-m_{2} \cos \varphi_{2}\right)- \\
& -m_{2} l_{2}\left(\dot{\varphi}_{2}\right)^{2}+k_{12} \sin \varphi_{2}\left(l_{2} \sin \varphi_{2}-l_{1} \sin \varphi_{1}\right)=0 .
\end{aligned}
$$

From the physical point of view, the described energy exchange can be considered as nonlinear targeted energy transfer [3-6]. The nonlinearity of the system is due to the time dependence of the string of the pendulum 2, which is tightly bound through the common string with particle 3 (which moves only vertically). The initial conditions correspond to the impact excitation of particle 1 in the pendulum 1 , which is weakly coupled to particle 2 through the spring with constant $k_{12}$. The string of pendulum 1 is shorter than the initial string of pendulum $2, l_{1}<l_{20}$. The masses of pendulums 1 and 2 are equal, $m_{1}=m_{2}$, while the mass of the particle 3 is larger, $m_{3}>m_{2}$. The particles 2 and 3 are initially at rest. Because of $l_{1}<l_{20}$ and $m_{3}>m_{2}$, initially non-resonant pendulums 1 and 2 pass through the internal resonance due to slow (adiabatic) shortening of the string of pendulum $2 l_{2}(t)$. The adiabatic sweep through the internal resonance results in irreversible transfer of vibration energy from pendulum 1 to pendulum 2. At this stage, the irreversible energy transfer between pendulums 1 and 2 reveals the vibration analogue of the nonadiabatic Landau-Zener tunneling, which has been predicted and experimentally confirmed in a system of two coupled parametrically driven pendulums $[7,8]$. But the centrifugal kinetic energy of pendulum $2,0.5 m_{2} l_{2}^{2}\left(\dot{\varphi}_{2}\right)^{2}$, stops the shortening of its string at some moment and the string length starts to increase, up to the initial length $l_{20}$. At this moment the total (cumulative) vibration energy of particles 2 and 3 returns back to pendulum 1, and particles 2 and 3 are again at rest.
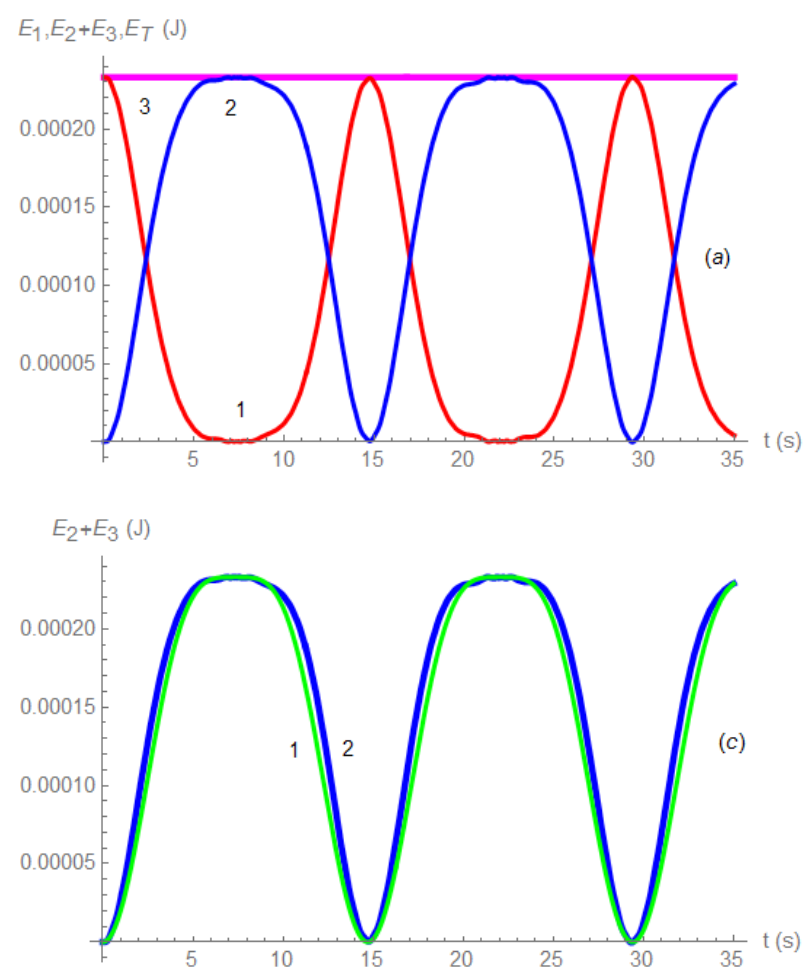

Fig. 1. (Color online) Time dependences of energy of pendulum $1 E_{1}$, red lines 1 in (a) and 2 in (b), of cumulative energy of particles 2 and $3 E_{2}+E_{3}$, blue lines 2 in (a) and (b), and of total energy $E_{T}=E_{1}+E_{2}+E_{3}$, magenta lines 3 in (a) and (d). Green lines 2 and 1 in (b) and (c) show the analytical prediction given by Eqs. (5) and (6), respectively. Lines 1 and 2 in (d) show the time dependences of the energies of particles 2 and 3 separately.

\section{Results and discussion}

In Fig. 1(a) we show the time dependences of the energy and $3, E_{2}+E_{3}$, and the total conserved energy of the system, $E_{T}=E_{1}+E_{2}+E_{3}$. In our simulations, we use the following initial conditions and parameters (which are close to that which were used in simulations and experimental setup in Ref. [8]): $l_{1}=0.305 \mathrm{~m}, l_{2}(0)=l_{20}=0.375 \mathrm{~m}, \dot{l}_{2}(0)=0$, $m_{1}=m_{2}=0.244 \mathrm{~kg}, m_{.3}=1.003 m_{2}, k_{12}=0.99 \mathrm{~N} / \mathrm{m}, \varphi_{1}(0)=0$, $\varphi_{2}(0)=0, \dot{\varphi}_{2}(0)=0, \dot{\varphi}_{1}(0)=0.143 \mathrm{rad} / \mathrm{s}$.

The time evolution of the energies $E_{1}$ and $E_{2}+E_{3}$ can be approximated with high accuracy by the parameterization of the three-state atomic system in a coherent superposition of the trapped and out-coupled states, cf. Ref. [2]:

$$
\begin{gathered}
E_{1}=0.000233 \cos ^{4}\left(\omega_{R} t / 2\right), \\
E_{2}+E_{3}=0.000233\left[\sin ^{4}\left(\omega_{R} t / 2\right)+\frac{1}{2} \sin ^{2}\left(\omega_{R} t\right)\right],
\end{gathered}
$$

where $\omega_{R}$ is the frequency of the Rabi-like coherent beats, which is determined by the coupling constant $k_{12}$ between pendulums 1 and 2,

$$
\omega_{R} \approx \frac{k_{12} l_{20}}{2 m_{1} \sqrt{g l_{1}}}=0.440 \mathrm{~s}^{-1},
$$

and is equal in our simulations to $0.427 \mathrm{~s}^{-1}$. On the other hand, our system operates in the double-resonance conditions when the frequency $\omega_{R} / 2$ coincides with the characteristic frequency $\omega_{1}=\pi /\left(2 t_{l}\right)$ of the periodic shortening and elongation, from $l_{20}$ to almost zero during $t_{l}$, of the string $l_{2}$ of pendulum 2:

$$
\omega_{l}=\frac{\pi}{2 t_{l}}=\pi \sqrt{\frac{g\left(m_{3}-m_{2}\right)}{2 l_{20}\left(m_{2}+m_{3}\right)}} \equiv 0.220 \mathrm{~s}^{-1} .
$$
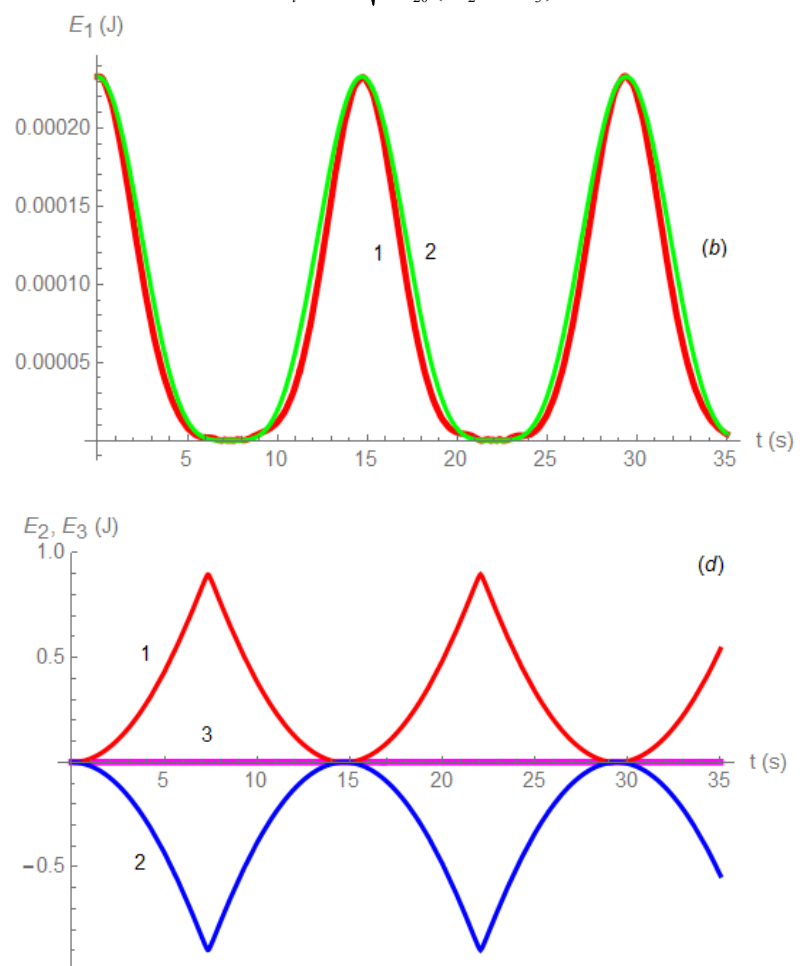
of pendulum $1, E_{1}$, the cumulative energy of particles 2 
In Fig. 1(d) we show the time dependences of the energies of particles 2 and 3 separately. Since energies in all plots are shown in absolute (SI) units, one can see that the magnitudes of maximal energies of particles 2 and 3 are larger than maximal energy of pendulum 1 in a proportion close to 4000:1! As one can also see from Figs. 1(a) , 1(b) and 1(c), so high ratio between the total and the given to pendulum 1 energies does not destroy the coherence of the system for the long time. Doubling of the initial deflection angle velocity of pendulum $1, \dot{\varphi}_{1}(0)$, changes only the ratio between the maximal energies of particles 2 and 3 and the maximal energy of pendulum 1, making it close to 900:1, but almost does not change the characteristic time dependences and periods of energy transfer. Further increase of $\varphi_{1}(0)$ results in the gradual enhancement of weak randomness of the nonlinear energy transfer between the subsystems, which is caused by the faster loss of coherence.

The described effects can be used for the designing of the devices, which can serve as controllable sources of coherent pulses of mechanical energy on the micro- and nano-scales. These effects can also be used for the "mechanical cooling" of a single oscillator weakly attached to energetic mechanical system with variable parameters.

\section{References}

1. M. Holland, K. Burnett, C. Gardiner, J. I. Cirac, P. Zoller. Phys. Rev. A 54, 1757 (1996).

2. M.-O. Mewes, M. R. Andrews, D. M. Kurn, D.S. Durfee, C. G. Townsend, W. Ketterle. Phys. Rev. Lett. 78, 582 (1997).

3. P. Maniadis, G. Kopidakis, S. Aubry. Physica D 188, 153 (2004).

4. Yu. A. Kosevich, L. I. Manevitch, A. V. Savin. Journal of Physics: Conference Series 92, 012093 (2007).

5. Yu. A. Kosevich, L. I. Manevitch, A. V. Savin. Phys. Rev. E 77, 046603 (2008).

6. Yu. A. Kosevich, L.I. Manevitch, A. V. Savin. Journal of Sound and Vibration 322, 524 (2009).

7. Yu. A. Kosevich, L.I. Manevitch, and E.L. Manevitch. Physics-Uspekhi 53, 1281 (2010).

8. L. I. Manevitch, Yu. A. Kosevich, M. Mane, G. Sigalov, L.A. Bergman, A.F. Vakakis. International Journal of Non-Linear Mechanics 46, 247 (2011) . 